Questions vives

\section{Questions Vives}

Recherches en éducation

Vol. $7 n^{\circ} 17$ | 2012

Des usages des TIC à la certification des compétences numériques : quels processus de formation et de validation?

\title{
Kiêm, T., Science de gestion de l'éducation : Quelques problématiques théoriques et pratiques
}

Khoa học quản lý giáo dục : Một số vấn đề lý luận và thực tiễnHanoi : Édition de l'Éducation, 2008

\section{Nguyên Tân Dai}

\section{(2) OpenEdition}

\section{Journals}

\section{Édition électronique}

URL : http://journals.openedition.org/questionsvives/1062

DOI : 10.4000/questionsvives. 1062

ISBN : 978-2-8218-1394-6

ISSN : $1775-433 \mathrm{X}$

Éditeur

Université Aix-Marseille (AMU)

Édition imprimée

Pagination : 179-181

ISSN : 1635-4079

\section{Référence électronique}

Nguyên Tân Dai, «Kiêm, T., Science de gestion de l'éducation : Quelques problématiques théoriques et pratiques », Questions Vives [En ligne], Vol.7 n¹7 | 2012, mis en ligne le 15 novembre 2012, consulté le 22 septembre 2020. URL : http://journals.openedition.org/questionsvives/1062 ; DOI : https://doi.org/ 10.4000/questionsvives.1062

Ce document a été généré automatiquement le 22 septembre 2020.

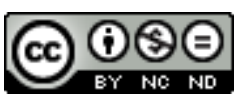

Questions Vives est mis à disposition selon les termes de la licence Creative Commons Attribution Pas d'Utilisation Commerciale - Pas de Modification 4.0 International. 


\section{Kiêm, T., Science de gestion de l'éducation : Quelques problématiques théoriques et pratiques}

Khoa học quản lý giáo dục : Một số vấn đề lý luận và thực tiễnHanoi :

Édition de l'Éducation, 2008

\section{Nguyên Tân Dai}

\section{RÉFÉRENCE}

Kiêm, T. (2008). Science de gestion de l'éducation : Quelques problématiques théoriques et pratiques. Hanoi : Édition de l'Éducation

\section{NOTE DE L'AUTEUR}

Trân Kiêm, est actuellement professeur en gestion de l'éducation à l'École Normale Supérieure de Hanoï au Vietnam. Il a été formé aux théories de l'enseignement en ex Union soviétique. Il est l'auteur de plus de 50 articles et 6 ouvrages (tous publiés en vietnamien) en sciences de l'éducation et plus particulièrement en gestion de l'éducation.

Dans cet ouvrage, Trân Kiêm, professeur à l'École Normale Supérieure de Hanoï, propose de revenir sur la naissance d'une nouvelle discipline de recherche dans le domaine de l'éducation au Vietnam. À partir d'une confrontation des approches théoriques dominantes avec la réalité des pratiques éducatives, l'auteur tente de dégager les éléments d'un nouveau modèle en science de gestion de l'éducation. 
2 Cet ouvrage, de trois cent pages, se compose de six chapitres précédés d'un avantpropos court rédigé par l'auteur lui-même, qui insiste sur l'intérêt de développer la discipline de science de gestion de l'éducation en dépit d'un contexte scientifique et économique défavorable.

3 L'auteur introduit le premier chapitre avec l'histoire des approches théoriques de gestion dans les sociétés traditionnelles en Asie et tout particulièrement en Chine et au Vietnam. Il présente ensuite de manière détaillée les principales théories de l'organisation en Occident qui se sont développées au cours du $20^{\text {ème }}$ siècle. Cet éclairage théorique permet à Trân Kiêm de poser dans le chapitre suivant les fondements d'une science de " gestion de l'éducation » qu'il considère comme l'activité consciente de l'homme pour atteindre ses objectifs. Dans un premier temps, il fait le lien entre l'approche conceptuelle de la "gestion» et les pratiques de terrain dans le domaine de l'enseignement avant de mettre au jour deux niveaux de gestion distincts pour le système éducatif : macro et micro. Dans un deuxième temps, l'auteur explique que la méthodologie de la science de gestion de l'éducation puise à deux sources: le marxisme-léninisme et les lois dialectiques. Ce deuxième chapitre est également l'occasion pour l'auteur d'insister sur plusieurs éléments: le caractère interdisciplinaire de la science de gestion de l'éducation, la nécessité de l'appliquer de manière réaliste et les trois fonctions de connaissance, d'amélioration et de prévision que doit remplir, selon lui, toutes les sciences.

4 Le troisième chapitre, qui représente le tiers de l'ouvrage, s'intitule « Le processus de gestion de l'éducation ». Ici, l'auteur défend l'idée que la gestion de l'éducation est un processus pour lequel il identifie les fonctions, les principes, les méthodes et les outils. L'analyse est démonstrative et s'appuie à la fois sur quelques approches de management développées en Occident et sur une idéologie du système de gestion sociale à la soviétique ou à la vietnamienne dite " orientation socialiste ».

5 Le quatrième chapitre est consacré à l'innovation de la gestion de l'éducation. Après avoir présenté les grandes tendances de l'éducation au niveau international, en se référant principalement aux déclarations de l'UNESCO faites dans les années 1990-2000, Trân Kiêm indique que l'innovation constitue l'un des enjeux majeurs de la gestion de l'éducation. Il présente ensuite une expérience menée dans une commune vietnamienne qui aurait donné "d'excellents résultats dans le secteur éducatif» et qu'il considère comme exemplaire pour l'ensemble de la communauté éducative du pays. La fin du chapitre, qui décrit quelques méthodes de gestion dans le champ professionnel (Total Quality Management) et éducatif (School-Based Management), s'adresse tout particulièrement aux gestionnaires de l'éducation qui peuvent s'en inspirer dans leur activité.

6 Le cinquième chapitre est très court et s'intitule «Travail de gestion de l'éducation et efficacité du travail de gestion de l'éducation ».Trân Kiêm explique brièvement le rôle du travail de gestion dans le domaine éducatif qu'il tente de caractériser avant de définir ce qu'il entend par " efficacité du travail de gestion de l'éducation ».

7 Dans le sixième et dernier chapitre, l'auteur évoque la situation critique de l'état de la recherche dans le domaine de la gestion de l'éducation. La consolidation d'une science de gestion de l'éducation est d'autant plus importante qu'elle peut constituer un véritable phare pour les acteurs du système éducatif. Pour Trân Kiêm, la science de gestion de l'éducation, émergente au Vietnam, s'inscrit dans un modèle triangulaire qui associe trois pôles plus ou moins rapprochés: l'innovation du travail de gestion, la 
recherche scientifique et la formation des professionnels en gestion de l'éducation. Pour conclure, l'auteur donne quelques indications générales à l'attention de tous les jeunes chercheurs qui souhaitent rédiger un rapport scientifique.

8 Au terme de cette lecture, dont nous avons tenté de rendre compte un peu rapidement, il apparait que l'intérêt de cet ouvrage réside principalement dans son objet d'étude. Au Vietnam, la science de gestion de l'éducation est considérée comme une nouvelle discipline ouvrant sur un domaine de recherche peu connu et peu étudié. C'est d'ailleurs ce qui rend le travail de l'auteur difficile lorsqu'il expose les fondements théoriques de cette discipline de recherche. A ce propos, on regrettera le style souvent laborieux de ce texte qui présente, au demeurant, de nombreuses lacunes.

D'abord, il manque un fil conducteur dans l'analyse que propose l'auteur. Les idées ne sont pas toujours articulées. Par exemple, on ne trouve aucune transition entre les différents chapitres ni mêmes les sous parties. Ensuite, tout au long de l'ouvrage, l'auteur juxtapose différentes approches de la notion de gestion de l'éducation, ce qui ne lui permet pas de dégager les aspects fondamentaux d'une telle science. Enfin, la revue de littérature s'appuie sur une bibliographie insuffisamment fournie. A peine une trentaine de documents sont référencés dont deux tiers relèvent des champs de la philosophie ou de la gestion administrative. Le tiers restant se rapporte plutôt à des textes législatifs et réglementaires plus ou moins associés au domaine de l'éducation.

$\mathrm{Au}$ fond, Trân Kiêm présente ici plus une réflexion philosophique sur la gestion de l'éducation que les fondements d'une véritable science. Il reconnaît d'ailleurs lui-même que le manque de publications scientifiques au Vietnam dans ce domaine mais aussi l'absence de liens avec la communauté de chercheurs en sciences de l'éducation au niveau international altèrent son travail d'analyse. Si le recours au mode de raisonnement idéologique est fréquent dans le monde de la recherche vietnamien, on regrettera également le manque d'objectivité de la part de l'auteur qui renforce l'impression générale d'une théorisation simpliste de ses expériences personnelles.

11 Bien que cet ouvrage comporte de nombreuses faiblesses, à la fois méthodologiques et théoriques, il faut reconnaître l'effort d'ouverture dont fait preuve l'auteur lorsqu'il tente quelques liens avec des théories modernes occidentales de gestion administrative ou éducative. A ce mérite peut s'ajouter celui d'avoir affirmé la nécessité de repenser la gestion de l'éducation en rapprochant expériences réelles et études théoriques.

\section{AUTEURS}

\section{NGUYÊN TÂN DAI}

Centre d'examen et d'évaluation de la qualité de formation, Université Nationale du Vietnam à Hô Chi Minh-Ville 